ELMANSORI, Ashraf, LORDING, Timothy, DUMAS, Raphaël, ELMAJRI, Khalifa, NEYRET, Philippe, LUSTIG, Sebastien, 2017, Proximal tibial bony and meniscal slopes are higher in ACL injured subjects than controls: a comparative MRI study, Knee Surgery, Sports Traumatology,

Arthroscopy, 25, 5, Springer Verlag, pp. 1598-1605, DOI: 10.1007/s00167-017-4447-4

\title{
1. PROXIMAL TIBIAL BONY AND MENISCAL SLOPES ARE HIGHER IN 2. ACL INJURED SUBJECTS THAN CONTROLS. A COMPARATIVE MRI 3. STUDY.
}

\section{ABSTRACT}

5. PURPOSE: Increased tibial slope is reported as a risk factor of non-contact anterior

6. cruciate ligament $(A C L)$ injury, but the effect of the soft tissues on slope remains

7. unclear. The primary aims of this study were to compare the tibial bony and soft tissue

8. slopes between patients with and without ACL injury, and to investigate the

9. relationship between the meniscal slopes (MS) and the tibial bony slope. Our

10. hypothesis was that the menisci would correct the inclination of the bony tibial slope

11. towards the horizontal.

12. METHODS: Using Magnetic resonance imaging (MRI), the lateral \& medial tibial slopes

13. (LTS, MTS) and lateral \& medial meniscal slopes (LMS, MMS) were compared in 100

14. patients with isolated $A C L$ injury and a control group of 100 patients with patello-

15. femoral pain and an intact ACL.

16. RESULTS: Repeated measures analysis of variance showed good inter- \& intra-observer

17. reliability for both bony and soft tissue slopes (ICC (0.88-0.93) and (0.78- 0.91$)$ for intra-

18. \& inter-observer reliability, respectively). The LTS \& MTS were significantly greater in

19. the $A C L$ injury group $(\mathbf{1 0 . 4 \pm 3 . 1 ~ \& ~ 9 . 4 \pm 3 . 3 ) ~ t h a n ~ i n ~ t h e ~ c o n t r o l ~ g r o u p ~} \mathbf{( 7 . 3 \pm 3 . 4 ~ \& ~}$ 
20. 7.0 $\$$ 3.7). Similarly, the LMS \& MMS were significantly greater in the ACL injury group

21. (4.7 $\pm 4.7 \& 6.0 \pm 3.4)$ than the control group $(0.9 \pm 4.8 \& 3.7 \pm 3.6)$. In both

22. groups, the lateral bony tibial slope was greater than the medial bony tibial slope, but

23. the medial soft tissue slope was greater than the lateral soft tissue slope.

24. CONCLUSION: Increased tibial slopes, both bony and meniscal, are risk factors for $\mathrm{ACL}$

25. injury. As the meniscus tends to correct the observed slope towards the horizontal, loss

26. of the posterior meniscus may potentiate this effect by increasing the functional slope.

27. LEVEL OF EVIDENCE: Level III.

28. KEYWORDS: Knee, Tibial slope, Anterior Cruciate Ligament Injury, Meniscus, MRI.

\section{INTRODUCTION:}

30. ACL injury occurs predominantly via non-contact mechanisms. Due to the high

31. incidence of long-term sequelae of $A C L$ injury, including pain, instability, and the early

32. development of osteoarthritis, identification of risk factors for ACL injury is an important

33. step in the development of injury prevention (33).

34. Recently in the literature, there has been a great focus on anatomic risk factors.

35. Posterior Tibial slope (PTS) is commonly defined as the angle between a line fit to the

36. posterior-inferior surface of the tibial plateau and a tibial anatomic reference line (14,

37. $15,17,18,23,30,32$.

38. Biomechanically, a higher tibial slope in the presence of a compressive load will 
39. generate a higher anterior shear component of the tibio-femoral reaction force,

40. resulting in increased anterior motion of the tibia relative to the femur. Because the $A C L$

41. is the primary restraint against this type of motion in the knee, it logically follows that

42. an increase in the posterior tibial slope will generate an increased load in the ACL. This

43. hypothesis was first noted by Butler in 1980 (4).

44. Reliable clinical measurements of posterior tibial slope are important for understanding

45. $A C L$ injury mechanisms. It is widely mentioned in the literature that $A C L$-injured

46. individuals have a greater posterior tibial slope than healthy controls (20).

47. It remains unclear whether the risk of non-contact $A C L$ injury is increased in

48. those with increased slope in one or both compartments, and individual analysis of the

49. compartments separately could be essential to understand the functional consequences

50. of tibial slope (21).

51. The medial and lateral PTS are not necessarily identical in one given knee and

52. differences of as much as $27^{\circ}$ have been reported in cadaveric studies (16).

53. Various models for PTS measurement on conventional lateral radiographs have been

54. described, however it is still imprecise. Due to superimposition of the hemi-plateaus,

55. the lateral tibial plateau is difficult to identify, and separate assessment of the plateaus

56. is not reliably possible on lateral radiographs (16).

57. Previous studies have validated different modalities for measuring posterior tibial slope

58. (20). No significant difference exists between radiographs, computed tomography, and

59. magnetic resonance imaging (MRI); recent work has focused on $M R I(20,29)$. Although

60. lateral radiographs are better to assess the medial PTS, they are inadequate for reliable 
61. and separate PTS and meniscal slope (MS) assessment (16). Therefore, it is

62. recommended to use conventional MRI scans of the knee, as they allow simple

63. assessment of each plateau separately, and provide the possibility to assess the MS

64. reliably (15), while methods using three-dimensional computed reconstructions are

65. time-consuming and complex (12).

66. The soft tissues (e.g. cartilage and meniscus) play a role in antero-posterior stability of

67. the knee joint and may influence the functional tibial slope. The posterior

68. horn of the menisci is thicker than the anterior horn, and this could decrease the

69. postero-inferior slope (19).

70. This study aims to evaluate the correlation between the tibial slope and non-

71. contact $\mathrm{ACL}$ - injury using $\mathrm{MRI}$, as well as to determine the effects of the menisci on

72. tibial slope.

73. It was hypothesized that the meniscus would reduce the differences in slope

74. between the medial and lateral compartments of the same knee. In addition, it

75. was hypothesized that the presence of the meniscus would correct the

76. inclination of the bony tibial slope towards the horizontal.

\section{MATERIALS AND METHODS:}

78. All patients referred to the Croix-Rousse Hospital between 2012 and

79. 2015 for consideration of knee interventions were eligible for

80. inclusion in the study. The inclusion criteria were an MRI scan, with adequate quality

81. images available on the Picture Archiving and Communication (PACS) system and 
82. a report detailing the status of the ACL. Exclusion criteria were osteoarthritis, patients

83. with open physes or under the age of 18 years, MRI evidence of intra-articular

84. pathology besides $A C L$ tear, prior surgery to the involved knee, and those where the

85. scanned length of the tibia was insufficient for calculation of the tibial axis.

86. Two groups of patients were thus established. The study group consisted of 100

87. patients ( 67 males \& 33 females) with MRI confirmed isolated partial or complete

88. rupture of the ACL. Patient age in this study group ranged from 18- 63 (Mean $\pm S D$,

89. $33.7 \pm 10.8)$. The control group consisted of 100 patients whose major complaint was

90. patello-femoral pain (52 males \& 48 females), and whose MRIs revealed an intact ACL.

91. Patient age in this control group ranged from $18-86$ (Mean $\pm S D, 43.6 \pm 15.9$ ).

92. MRIs were obtained from a single 1.5-T MRI scanner (manufacturer-supplied

93. quadrature head coil, Philips Medical Systems). Proton density sagittal slices were used

94. to measure the angles. All measurements of the bony tibial slope and meniscal slope

95. angles were carried out using the annotation tools on the PACS provided by the

96. hospital, which allows tracing of the anatomical landmarks, connecting regions of

97. interest, and measurements of different angles. We chose three sagittal images from

98. the corresponding axial cuts at the joint line for three different cut regions: the mid-

99. sagittal cut, which use for calculating the proximal tibial anatomic axis (PTAA) (Figs.

100. 1\&2), the mid-lateral tibial plateau cut (LTP) (Figs. $\mathbf{3}, \mathbf{4} \& 5)$, and the mid-medial tibial

101. plateau cut (MTP) (Figs. 6, 7\&8).

102. Two independent reviewers, blinded to subjects' details, calculated the angles on each

103. MRI using a modified Hashemi method described previously by Lustig et al (21). 
104. The first observer performed two sets of measurements for each subject, two weeks

105. apart. The second observer, blinded to the results of the first, repeated the

106. measurements in a random specimen order.

107. To establish the tibial slope, we used PTAA which demonstrates the best correlation

108. with the tibial shaft anatomic axis (TSAA) (6). The PTAA is described on the mid-sagittal

109. cut by a line joining the midpoint between the anterior and posterior tibial cortices at

110. the level of the tibial tuberosity and at another level $5 \mathrm{~cm}$ more distal. The angle

111. between the tibial axis and the horizontal was then calculated (PTTA-H

112. Angle). This angle was used to transfer the PTAA among different images.

113. The MTP and LTP cuts were used to measure the medial and lateral tibial slopes (MTS,

114. LTS) respectively. The PTAA was superimposed onto these cuts by means of the PTTA-H

115. angle. The tibial slope in each compartment was measured as the angle between a line

116. connecting the highest points of the anterior \& the posterior parts of the tibial plateau,

117. and a line perpendicular to the PTTA. A posterior inclination to the line perpendicular

118. to the PTAA was assigned a positive value, while an anterior inclination was assigned a

119. negative value.

120. All measurements were positioned as an overlay and remained in a fixed position on

121. the complete image series. The lateral meniscal slope (LMS) and medial meniscal slope

122. (MMS) were defined in the same manner as the PTS. A line joining the superior edge of

123. the meniscosynovial border of the anterior and posterior horns of the meniscus in the

124. sagittal plane was chosen instead of the tibial plateau cortex.

\section{STATISTICAL ANALYSIS:}


FULL TEXT

126. The PTS and MS angles were reported as mean angles with standard deviations.

127. Data were statistically analyzed using SPSS for Windows statistical package

128. (version 11.5; SPSS, Chicago, IL, USA). The assumption of normality was assessed with

129. Kolmogorov-Smirnov tests. Intra-observer \& inter-observer reliability was tested by

130. means of Intra class Correlation Coefficient (ICC) to establish whether the mean slope

131. was altered between repeated measurements of the two observers.

132. Independent two sample t-test was used to compare the four variants between the two

133. groups. We calculated a sample size of 95 subjects was required, to achieve $90 \%$ power

134. to reject the null hypothesis (alpha $=0.0001)$.

\section{RESULTS:}

136. The test of normality revealed that all measured parameters for both groups were

137. normally distributed $(p<0.0001)$. The data were initially analyzed for each reviewer

138. and then the parameters were compared between the two groups.

139. Intra-observer \& inter-observer reliability:

140. The inter-observer ICC for all variants of the $A C L$ group ranged

141. from 0.89 to 0.91 and for the control group from 0.87 to 0.90 . Similarly, the

142. intra-observer ICC was high (0.88-0.93) which reveal strong agreement between

143. observers for all measurements.

144. The means, standard deviations (SD) and ranges for the repeated measurements LTS,

145. MTS, LMS, and MMS for the examined and control groups are shown in the table (1).

146. For the ACL injury group, the LTS ranged from $1.9^{\circ}$ to $15.8^{\circ}$ and the MTS ranged 
147. from $1.4^{\circ}$ to $16^{\circ}$ while the LMS ranged from $-5.6^{\circ}$ to $13.8^{\circ}$ and the MMS ranged from

148. $-2.2^{\circ}$ to $14.5^{\circ}$. Similarly, for the control group the LTS ranged from $0^{\circ}$ to $15.4^{\circ}$, and

149. the MTS ranged from $-0.6^{\circ}$ to $15.0^{\circ}$ while the LMS ranged from $-12.5^{\circ}$ to $12.6^{\circ}$ and

150. the MMS ranged from $-4.2^{\circ}$ to $11.5^{\circ}$. In the analysis of the ACL injury group the LTS

151. was larger than the MTS but the LMS was smaller than MMS. In the control group, the

152. LTS was also larger than the MTS and the LMS was smaller than MMS (fig.9).

\section{Comparison of the variants of the two groups:}

154. By direct comparison of the two groups, using independent a two sample student's t-

155. test, the LTS \& MTS were significantly greater in the ACL injury group than the control

156. group ( $p$ was 6.76 for LTS \& 4.85 for MTS). Similarly, the LMS \& MMS were significantly

157. greater in the ACL injury group than the controls ( $p$ was 5.67 for LMS \& 4.61 for

158. MMS).

\section{DISCUSSION:}

160. The most important finding of this study is that the lateral and medial bony and soft

161. tissue slopes were all significantly higher in the ACL injured group compared to the

162. control group. In both groups, the lateral bony tibial slope was greater than the medial

163. bony tibial slope, but the medial soft tissue slope was greater than the lateral soft

164. tissue slope. These findings are generally consistent with the published literature

165. (Table 2). 
166. Regarding the bony slope, Brandon et al compared the radiographic posterior

167. inferior tibial slope, as measured on plain radiographs, between $\mathrm{ACL}$ injured

168. subjects and controls (2). They found an increased slope in the ACL injured group,

169. and that within the $A C L$ injured group, increased slope correlated with increased

170. severity of the pivot shift. Stijak et al, using radiographs to establish the PTAA and then

171. MRI to measure the bony slope in each compartment, found an increased lateral bony

172. slope in ACL injured subjects (24). Hashemi et al, in an MRI study, found increased

173. lateral tibial plateau slope and decreased medial plateau concavity in the ACL injured

174. group in both sexes, as well as an increased medial slope in ACL injured males (13).

175. Similar results have also been reported in the pediatric population $(6,22)$. Not all

176. studies have supported these findings. Blanke et al, in a study of recreational skiers,

177. found no difference in the lateral or medial slope, or medial plateau concavity, between

178. ACL injured and non-injured subjects (1). Hudek et al examined not only the bony tibial

179. slopes, but also the influence of the menisci or the soft tissue slope (15). In contrast to

180. the aforementioned studies, they found no difference between ACL injured and

181. healthy controls with regards to the bony lateral and medial slopes. However, they

182. found increased lateral meniscal slope in the ACL injured group. Lustig et al examined

183. the effect of the meniscus on the observed slope in non-ACL injured subjects (21). In

184. line with the findings of our study; they found the meniscal slope to be more horizontal

185. than the bony slope in both compartments, with the lateral meniscal slope closer to

186. horizontal than the medial meniscal slope. Bonnin and Dejour investigated the impact

187. of the tibial slope on knee biomechanics, they found a $6 \mathrm{~mm}$ increase in anterior 
188. translation for each $10^{\circ}$ increase in slope in healthy and $A C L$ deficient knees (8). In a

189. cadaveric study, Giffin noted an increase in anterior tibial resting position with

190. increased tibial slope that was accentuated under an axial load (10).

191. The effects of patient demographics, such as gender and age, on the tibial slope have

192. not been fully elucidated. Females are at greater risk of non-contact ACL injury and a

193. steeper tibial slope has been observed in females (21). Multiple studies have shown

194. that women have a greater propensity for ACL injury compared to their male

195. counterparts $(\mathbf{1 1}, \mathbf{1 3}, \mathbf{1 5}, \mathbf{2 4}, \mathbf{3 3})$. It has been suggested that a possible risk factor for

196. this observation is that women have a narrower notch than men and even smaller ACLs.

197. Differences in slope between the medial and lateral compartments may also have

198. biomechanical consequences. Under a compressive load, the lateral femoral condyle

199. has been postulated to slide posteriorly due to increased LTS, pivoting around the

200. medial femoral condyle. The resultant external femoral rotation has been shown to

201. place excess strain on the $\operatorname{ACL}(\mathbf{9}, \mathbf{2 3})$.

202. Increased posterior slope is a risk factor not only for an ACL tear, but also for failure of

203. ACL reconstruction. Webb et al found an increased risk of failure or contralateral injury

204. associated with increased tibial slope, which was most apparent with the posterior

205. slope in excess of $12^{\circ} \mathbf{( 3 1 )}$. Similarly, Christensen et al found increased lateral slope to

206. be associated with a risk of early failure, regardless of graft type (5). Slope levelling

207. osteotomy has been proposed to correct this risk factor in repeat revision surgery (9, 208. 28).

209. The importance of the meniscal contribution to slope and knee biomechanics remains 
210. unclear. While Lustig demonstrated more horizontal meniscal slopes when compared

211. to the bony slopes, this MRI based study could not evaluate the effect of joint loading

212. and meniscal mobility (21). Song has defined the abnormal lateral plateau slope as a

213. risk factor for a high grade pivot shift (27). Loss of the posterior lateral meniscal root

214. also increases lateral compartment anterior translation during a pivot shift maneuver

215. (25). Under load, loss of the meniscal root could convert the effective functional slope

216. from the meniscal slope to the bony slope, which may explain these findings. Song, in a

217. separate study, also found increased medial meniscal slope to be a risk factor for ramp

218. lesions, particularly when time to surgery was greater than six months (26).

219. An understanding of the anatomical risk factors for $A C L$ injury and treatment failure is

220. important. While some factors, such as the width of the intercondylar notch, are not

221. readily modifiable, their identification may help to target proven prevention strategies

222. towards high risk individuals, in the same manner in which they are targeted towards

223. high risk sports. As a risk factor, increased tibial slope is potentially modifiable by

224. osteotomy. Such intervention should be considered in cases of excessive slope,

225. particularly after the loss of the menisci; however, the appropriate threshold for

226. intervention remains unclear.

227. One of the greatest strengths of using MRI for this application is the ability to visualize

228. the surface geometry of the articular cartilage, which represents the functional point of

229. the tibiofemoral articulation. Our study presents a reliable method for

230. measurement of the tibial and meniscal slopes in both knee compartments, which

231. could be developed as a predictive tool for ACL risk. The aims of future research 
232. relating PTS to $A C L$ injury risk should be to establish not only the extent of the role of

233. tibial slope in injury, but also to which that risk can be minimized by preventive

234. measures such as neuromuscular training (33).

235. This study is subject to a number of limitations. Firstly, all MRI scans were performed in

236. a recumbent position. Weight bearing may affect the meniscal height both anteriorly

237. and posteriorly and thus alter the measured meniscal slope. Furthermore, the control

238. group consisted of patients with patello-femoral pain, but no intra-articular pathology

239. as seen on MRI scans. While a similar group has been used in multiple previous studies

240. $(\mathbf{2}, \mathbf{1 5}, \mathbf{2 1}, \mathbf{2 4})$, these patients may in fact have tibial slope characteristics that differ

241. from a true asymptomatic population.

\section{CONCLUSION}

243. This study confirmed that the tibial bony \& soft tissue slopes can be measured reliably

244. using an MRI-based method. Increased tibial slopes, both bony and meniscal, are risk

245. factors for ACL injury. As the meniscus tends to correct the observed slope towards the

246. horizontal, loss of the posterior meniscus may potentiate this effect by increasing the

247. functional slope.

\section{Competing interests:}

249. The authors declare that they have no competing interests. 